\title{
The second English edition of the Japanese Classification of Gastric Carcinoma. A Western commentary
}

\author{
Paul Hermanek \\ Department of Surgery, University of Erlangen, Krankenhausstr.12, D-91054, Erlangen, Germany
}

To the Editor: After publication of the new edition of the Japanese classification of gastric carcinoma [1], three important questions arise for Western people:

1. Does the new edition contribute to a harmonization between the International Union Against Cancer (UICC) [2] and the Japanese classification of anatomic extent of carcinoma?

2. Are the new rules for the description of histomorphology in agreement with the WHO classification [3] as generally accepted in Western countries?

3. Are the rules for description of surgical treatment acceptable in Western countries?

These questions will be discussed below, especially considering the importance of an internationally uniform language in describing diagnosis and treatment, which is an indispensable precondition of any worldwide comparison between institutions.

\section{Description and classification of anatomic extent}

General principles. In the new edition, the anatomic extent is described clinically, surgically, pathologically, and finally. For local spread $(\mathrm{T})$, regional lymph node $(\mathrm{N})$, peritoneal $(\mathrm{P})$, liver $(\mathrm{H})$, and other distant metastasis (M), the type of assessment is indicated by the prefixes $\mathrm{c}, \mathrm{s}, \mathrm{p}$ and $\mathrm{f}$, respectively. While in the former edition clinical assessment was described by capital letters, now cT, cN, cH, cP, and cM are used. For pathological assessment, the former $\mathrm{t}, \mathrm{n}, \mathrm{p}, \mathrm{h}$, and $\mathrm{m}$ have been replaced by $\mathrm{pT}, \mathrm{pN}, \mathrm{pP}, \mathrm{pH}$, and $\mathrm{pM}$. This corresponds to the UICC style. However, the UICC clinical classification summarizes the separate Japanese clinical and surgical classifications.

Local spread (T). For the classification of the local spread of the primary tumor, i.e., the depth of invasion, identical categories (T1-T4) are used in the UICC and in the Japanese classification. In the 2nd Japanese edi- tion, the subclassification of T1 and T2 was introduced as proposed by the UICC TNM Supplement 1993 [4], i.e., $\mathrm{T} 1 \mathrm{a}=\mathrm{T} 1 \mathrm{M}$ (mucosa, muscularis mucosae), $\mathrm{T} 1 \mathrm{~b}$ $=\mathrm{T} 1 \mathrm{SM}$ (submucosa), T2a = T2MP (muscularis propria), and $\mathrm{T} 2 \mathrm{~b}=\mathrm{T} 2 \mathrm{SS}$ (subserosa). According to the new Japanese edition, in patients treated by mucosal resection, submucosal carcinomas should be further subdivided into SM 1 (submucosal invasion less than $0.5 \mathrm{~mm}$ ) and SM 2 (submucosal invasion $0.5 \mathrm{~mm}$ or more), predominantly because longterm outcome data after endoscopic mucosal resections are not yet available.

Regional lymph nodes $(\mathrm{N})$. Now, as before, there are considerable differences in the classification of regional lymph nodes. First of all, in the Japanese classification the definition of regional lymph nodes is different according to the tumor localization, while in the UICC system no respective differentiation is provided. Secondly, some lymph node stations considered as regional at least for some tumor localizations by the Japanese rules $(13$, retropancreatic; $14 \mathrm{v}$, superior mesenteric vein; $16 \mathrm{a} 2$ and $16 \mathrm{~b} 1$, paraaortic middle) are always classified as distant by the UICC. In consequence, there are patients with lymph node metastases who are assigned to M0 in the Japanese system and to M1 in the UICC system.

Furthermore, the subclassification of node-positive patients into $\mathrm{N} 1, \mathrm{~N} 2$, and $\mathrm{N} 3$ follows different principles: in the UICC system, it is based on the number, and in the Japanese system, on the localization of the involved nodes.

A translation of the UICC $\mathrm{N}$ classification into the Japanese system is not possible because documentation of the site of involved regional lymph nodes is not provided. On the other hand, translation of the Japanese system into the UICC N classification is possible, if for the individual lymph node stations, the number of involved nodes is documented as required in the Japanese rules. 
Table 1. UICC versus Japanese stage grouping

\begin{tabular}{ll}
\hline UICC [2] & Japanese classification, 2nd English edition [1] \\
\hline IA & IA \\
IB & IB, II, or IV \\
II & IB, II, IIIA, or IV \\
IIIA & II, IIIA, IIIB, or IV \\
IIIB & IIIA, IIIB, or IV \\
IV & IB, II, IIIA, IIIB, or IV
\end{tabular}

UICC, International Union Against Cancer

It should be emphasized that the new edition of the Japanese rules includes a new subdivision of lymph node stations 11 (into 11p, proximal splenic; 11d, distal splenic) and 12 (12a, left; 12b, posterior hepatoduodenal). This new subclassification influences the $\mathrm{N}$ and $\mathrm{M}$ category for some tumor localizations; thus, patients classified according to the 1st English edition of the Japanese rules cannot be compared with those classified according to the new rules.

Distant metastasis. In the new edition of the Japanese classification, the subclassification of peritoneal and liver metastasis has been abandoned. This corresponds to the UICC system. There is now only a terminological difference: peritoneal metastasis is coded P1 in the Japanese system and M1PER in the UICC system, while liver metastasis is coded as $\mathrm{H} 1$ and M1HEP, respectively.

Stage grouping. Now, as before, the UICC and the Japanese stage groupings cannot be directly compared; only stage IA is identical (Table 1). The reasons are the different definitions of regional and nonregional lymph nodes and of the $\mathrm{N}$ categories.

Tumor localization. There are some differences between the Japanese system and the description by the Topography Section of the International classification of diseases for oncology, 2nd edition (ICD-O-2) [5] as used in Western countries (Table 2). Without doubt, the Japanese classification gives more detailed information. It not only describes the involvement of the three main parts more exactly, but also considers the involvement of the cross-sectional circumference (anterior and posterior wall, lesser and greater curvature). Further advantages are the newly introduced code for circumferential involvement ("Circ") and the new special description for carcinomas of the remnant stomach.

\section{Description of histomorphology}

Now, as before, there are major differences between the Japanese description of histomorphology and the WHO classification as generally used in Western countries. The main differences relate to typing and grading.
Table 2. Description of tumor localization: ICD-O-2 versus Japanese classification (2nd English edition)

\begin{tabular}{lll}
\hline ICD-O-2 [5] & & Japanese classification [1] \\
\hline Upper third & C16.0 (Cardia) & U \\
& C16.1 (Fundus) & U or UM \\
Middle third & C16.2 (Body) & M, ML, or MU \\
Lower third & C16.3 (Antrum) & L, LD, LM \\
& C16.4 (Pylorus) & L, LD \\
C16.8 (Overlapping) & LMU, MUL, MLU, UML
\end{tabular}

ICD-O-2, International classification of diseases for oncology, 2nd edition; $\mathrm{D}$, duodenum

In the WHO classification, the histomorphology of carcinomas is described by two separate categories, namely, histologic type and histologic grade of differentiation. In contrast, the Japanese system provides combined categories, e.g., well and moderately differentiated tubular adenocarcinoma or poorly differentiated adenocarcinoma, while for other types, such as papillary or mucinous adenocarcinoma, no grading is used.

Another difference relates to the grading of tumors with different grades of differentiation. In such cases, the final grade according to the WHO classification is determined by the highest grade, while in the Japanese system, the final grade is determined by the predominant pattern.

In summary, comparisons between the WHO typing and grading and the Japanese combined "histological typing" are, except for some cases, not generally possible.

Minor differences exist in regard to lymphatic and venous invasion. For lymphatic invasion, the UICC distinguishes two categories (L0, no lymphatic invasion; L1, lymphatic invasion), while the Japanese rules provide a subclassification of lymphatic invasion (ly 1 , minimal; ly 2, moderate; ly 3 , marked lymphatic invasion). However, the three Japanese categories are in no manner defined; thus subjective and non-reproducible assessing may be expected. The same comment is valid for the Japanese subclassification of venous invasion ( $\mathrm{v} 1$, minimal; v2, moderate; v3, severe venous invasion). In contrast, the UICC provides clearly defined categories (V0, no venous invasion; V1, microscopic; and V2, macroscopic venous invasion [including macroscopic involvement of the wall of veins with no tumor within the vein]).

\section{Description of surgical treatment}

From a Western point of view, the Japanese description of surgical treatment, as partly newly formulated in the 
2nd edition of the Japanese classification, includes three major problems.

Lymph node dissection. In Western countries, in most cases the so-called D classification distinguishes between D1, D2, and D3, with the following definitions: $\mathrm{D} 1$, dissection of perigastric nodes (stations 1-6); D2, additional dissection of the nodes of the gastric bed (stations 7-12); and D3, additional dissection of distant abdominal and/or mediastinal nodes (involvement classified as distant metastases, M1). These classifications are applied to tumors of any localization.

In contrast, the Japanese D classification is based on the grouping of node stations into lymph node groups (compartments) which are defined depending on tumor localization. Thus, any comparison between the simplified Western and the Japanese D classification is completely impossible.

Local completeness of gastrectomy (residual tumor or $\mathrm{R}$ classification). For gastrectomy, the Japanese classification provides only statements on the proximal and distal resection margins (PM, proximal; and DM, distal margin, corresponding to $\mathrm{OW}$ and $\mathrm{AW}$ in the former edition). In Western countries, however, it is obligatory to assess the circumferential (radial) resection margin, because this is the site where tumor at the margin is found in the majority of incomplete tumor resections [6]. Thus, according to the Japanese rules, an R classification according to the UICC cannot be performed.

Residual tumor $(\mathrm{R})$ classification versus classification of curative potential. In Western countries, the R classification with its highly significant prognostic significance [7] is generally considered as an indispensable variable in treatment result and prognostic factor analysis. In contrast, in Japan, resections with similar intent are classified according to the so-called curative potential (in the former edition, designated as curability). It has to be emphasized that a resection C in the Japanese classification corresponds to the Western R1 or R2; however, only some, and not all, R1 and R2 patients are classified as resection $\mathrm{C}$. The reason is that patients with tumor on the circumferential (radial) margins are not identified in the Japanese classification system, although the prognosis of these patients is identical to that of patients with tumor at the proximal or distal margin.

In the new edition of the Japanese classification, the definitions of categories for the curative potential of gastric resection have been changed in regard to the margins. In the former edition, resection (curability) A required margins " $>10 \mathrm{~mm}$ " on surgical evaluation and ">5 mm (histologically)" on conclusive (final) evaluation. In the new edition, resection A requires "proximal and distal margins $>10 \mathrm{~mm}$ " (without statements on the method of measurement). Thus, it remains doubtful whether the required " $>10 \mathrm{~mm}$ " relates to gross or to histologic measurement.

\section{Further perspectives}

The new English edition of the Japanese classification of gastric carcinoma includes some steps in the direction toward a harmonization with the UICC TNM classification of anatomic extent of cancer. However, considerable differences remain, in particular, in the definition of regional and distant lymph nodes, the classification of regional lymph node metastasis, and the stage grouping. Thus, direct comparisons between the two classification systems in regard to the $\mathrm{N}$ and $\mathrm{M}$ classification and the stage grouping are not possible. The same comment is valid for the Japanese description of histomorphology and the WHO typing and grading as generally used in Western countries. Lastly, a direct comparison is not possible in regard to the description of the immediate results of surgery, i.e., extent of lymph node dissection (D classification) and local completeness of gastrectomy (residual tumor or $\mathrm{R}$ versus curative potential classification).

The different systems of documentation and classification of gastric carcinoma patients make international comparisons of treatment results and worldwide multicenter analyses of data impossible. This explains some different results of therapeutic trials and studies of prognostic factors. Thus, a uniform international documentation system for gastric cancer (IDS for GC) is needed as a reporting system [8].

Such a system includes all variables, enabling a classification according to both Western and Japanese classification systems. It is an indispensable prerequisite for a methodically unobjectionable evaluation of the existing different classification systems, which requires the prospective comparison of the different systems in identical patients in high- and low-mortality countries. It seems an important task to organize worldwide efforts for the realization of such a comparative evaluation of the UICC/WHO and the Japanese classifications of gastric carcinoma.

\section{References}

1. Japanese Gastric Cancer Association. Japanese classification of gastric carcinoma. 2nd English ed. Gastric Cancer 1998;1:10-24.

2. UICC, Sobin LH, Wittekind Ch, editors. TNM classification of malignant tumours. 5th ed. Berlin Heidelberg New York Tokyo: Springer-Verlag; 1997.

3. Watanabe H, Jass JR, Sobin LH. Histological typing of oesophageal and gastric tumours. 2nd ed. WHO international histological classification of tumours. Berlin Heidelberg New York Tokyo: Springer-Verlag; 1990:1-2.

4. UICC, Hermanek P, Henson DE, Hutter RVP, Sobin LH, editors. TNM supplement 1993. A commentary on uniform use. Berlin Heidelberg New York Tokyo: Springer-Verlag; 1993:120-1.

5. WHO, Percy C, van Holten V, Muir C, editors. International classification of diseases for oncology. 2nd ed. Geneva: WHO; 1990:4. 
6. Hermanek P. pTNM and residual tumor classifications: problems of assessment and prognostic significance. World J Surg 1995;19:184-190.

7. Hermanek P, Maruyama K, Sobin LH. Stomach carcinoma. In: UICC, Hermanek P, Gospodarowicz MK, Henson DE, Hutter RVP, Sobin LH, editors. Prognostic factors in cancer.
Berlin Heidelberg New York Tokyo: Springer-Verlag; 1995:4763 .

8. Hermanek P. International documentation system for gastric carcinoma (IDS for GC): objectives and methods. In: Siewert JR, Roder JD, editors. Progress in gastric cancer research 1997. Bologna: Monduzzi Editore; 1997:1665-8. 